\title{
Is Advanced Paternal Age associated with Fetal Growth and Adverse Neonatal Outcomes? A Case-Control Study
}

\author{
${ }^{1}$ Manisha Dudlani, ${ }^{2}$ Tarakeswari Surapaneni \\ ${ }^{1}$ Fellow, High-Risk Pregnancy and Perinatology, Department of Obstetrics, Fernandez Hospital Pvt Ltd, Hyderabad, Andhra Pradesh, India \\ ${ }^{2}$ Consultant, Department of Obstetric Medicine, Fernandez Hospital Pvt Ltd, Hyderabad, Andhra Pradesh, India
}

Correspondence: Manisha Dudlani, Fellow, High-Risk Pregnancy and Perinatology, Department of Obstetrics, Fernandez Hospital Pvt Ltd, Hyderabad, Andhra Pradesh, India, e-mail: manisha_dudlani@hotmail.co.uk

\section{ABSTRACT}

Aim: To determine associations of advanced paternal age with fetal growth and adverse neonatal outcomes.

Methods: A hospital-based unmatched case-control study with random selection of controls. Fetal growth was determined by serial ultrasound measurements and growth was classified at birth by a neonatalogist based on the Lubchenco charts. Advanced paternal age was explored using two cutoffs ( 35 and 40 years). Likelihood ratios, unadjusted and adjusted odds ratios and the $95 \%$ confidence intervals around point estimates are presented.

Results: The study covered 218 pregnant women that included $137(63.72 \%)$ pregnant women who delivered a live AGA/LGA baby and $78(36.28 \%)$ pregnant women who delivered a live small for gestational age baby and $45(20.64 \%)$ fathers with advanced paternal age based on a cutoff of $\geq 40$ years and $73(33.49 \%)$ fathers with advanced paternal age based on a age cutoff $\geq 35$ years. Although advanced paternal age (both $\geq 35$ and $\geq 40$ years) was protective for small for gestational age babies in a bivariate analysis, the association was not significant in a multivariate regression model that adjusted for maternal age, parity, diabetes and gestational age at delivery. Advanced paternal age (both $\geq 35$ and $\geq 40$ years) did not show a clinically meaningful positive or negative likelihood ratio with other adverse neonatal outcomes.

Conclusion: Paternal age does not seem to be associated with fetal growth or adverse neonatal outcomes; however, a prospective cohort study is necessary to provide further evidence after controlling for potential confounders.

Keywords: Paternal age, Neonatal outcomes, Fetal growth, Small for gestational age.

\section{INTRODUCTION}

Studies have reported that advanced paternal age is associated with various adverse outcomes that include fetal loss, congenital malformations, single gene disorders, lower intelligence, dyslexia and mental disorders in children as well as low birth weight, preterm births or reduced growth. ${ }^{1-9}$ An association between advanced paternal age and adverse outcomes is biologically plausible through greater expression of paternal genes on placenta and the higher chances of mutations that involve those genes in men at extremes of age (young or advanced ages)..$^{10-12}$ Environmental conditions can hasten adverse biologic effects and may lead to a potential association between paternal age and adverse perinatal outcomes. ${ }^{13-15}$ An indirect association is also possible as paternal factors, especially those related to environment and risk behaviors, may lead to maternal exposure and an indirect additional effect on the fetus or alter the germ cell line or metabolic activity of the

Date of Received: 23-05-11

Date of Acceptance: 25-05-11

Date of Publication: September 2011 sperm leading to increased infertility or abnormality in conception. ${ }^{16-18}$

Demographic and lifestyle shifts have resulted in an increasing age at conception (natural or assisted) with 'older' mothers no longer a rarity and a consequently, a possible increase in paternal age at conception. We designed a case control study to determine if paternal age was independently associated with fetal growth (primary outcome) and adverse neonatal outcomes in a cohort of pregnant women at an advanced tertiary care center in India.

\section{METHODS}

The study protocol, which adhered to the tenets of the declaration at Helsinki, was approved by the Institutional Review Board of Fernandez Hospital at Hyderabad, India. Details of each pregnancy for each woman who has undergone antenatal care at Fernandez Hospital are entered into an electronic database. These include a detailed clinical examination routine with collection of demographic details, past and current obstetric and medical history, clinical examination and investigation protocols, ultrasound screening for structural abnormalities and nuchal translucency at 11 to $13^{+6}$ weeks, 
screening for gestational diabetes, targeted ultrasound screening in the second trimester for fetal abnormalities (TIFFA scan) and fetal well being scans in the third trimester.

Ultrasound examinations for serial growth measurements were performed by trained obstetricians with a Voluson 730 Expert machine with curved array transabdominal transducer AB 2 to $7 \mathrm{MHz}$ with multihertz and harmonic capability (GE Medical system, Kretz ultrasound) and HP machine with transabdominal probe. All ultrasound images were stored in digital imaging media (DICOM, SONOCARE) and available for retrospective assessment and remeasurement of all fetal parameters as needed. The variables used to measure growth were biparietal diameter (BPD), head circumference (HC), abdominal circumference (AC) and femur length (FL). The combination of all variables was used to derive the estimated fetal weight (EFW) and growth pattern of the given fetus. The population-based growth curves from Sonocare software developed by Mediscan systems, Chennai on a South Indian population were used to determine the type of fetal growth. Fetuses whose growth parameters fall in between 5 th and 95th centile were categorized as average for gestational age (AGA), fetuses whose growth parameters fell below 5 th centile or who were showing static growth over a period of time were considered as growth restricted fetuses (FGR), fetuses whose growth parameters fell on 5 th centile but showed a good interval growth were considered as reduced growth fetuses and a fetus whose growth was greater than the 95 th centile was considered large for gestational age (LGA). After birth, the neonate was categorized as small for gestational age, appropriate for gestational age or large for gestational age by the neonatologist using Lubchenco charts.

We defined a case as a fetus with restricted growth determined by ultrasound in the third trimester of pregnancy and confirmed by neonatal examination as a SGA baby immediately after birth. We defined a control as an AGA/LGA fetus determined by ultrasound in the third trimester of pregnancy and confirmed by neonatal examination immediately after birth.

The study included pregnant women who were booked for antenatal care prior to 20 weeks at Fernandez Hospital, had a singleton fetus, had ultrasound dating of pregnancy, third trimester scan for fetal wellbeing and delivered a live baby. Pregnant women with multiple pregnancies or those who were booked for antenatal care after 20 weeks of gestation were not considered for inclusion. The sample size for an unmatched case-control study was estimated for a ratio of two controls per case, $80 \%$ power, $95 \%$ confidence, and a hypothetical proportion of exposure (SGA) in those without advanced paternal age of $25 \%$. Using these parameters, 69 cases and 137 controls were sufficient to detect a least extreme odds ratio of 0.28 . Medical records in the database for the period January 2010 to September 2010 were explored to identify cases and controls from the eligible pool. Controls were selected randomly using a simple random selection strategy from the list of live AGA babies born on the same day as the cases to minimize selection bias. Advanced paternal age was defined and divided into two groups based on two different age cutoffs ( $\geq 40$ years and $\geq 35$ years).

Data was entered into a MS Office, Excel spreadsheet and exported into SPSS for Windows (version 16.0) statistical software package for analysis. We used the Chi-square test and Fishers Exact test to compare categorical variables and an analysis of variance (ANOVA) test to compare the means of continuous variables between the cases and controls. The association of neonatal outcomes stratified by advanced paternal age was initially explored using a bivariate analysis and termination of an unadjusted odds ratio and $95 \%$ confidence intervals around the odds ratio. We also estimated an adjusted odds ratio (adjusted for factors found significantly different between the cases and controls) 95\% confidence intervals around the odds ratio using a multivariate analysis to determine associations of paternal age with fetal growth and adverse perinatal outcomes. A p-value $<0.05$ was considered statistically significant. We also used the likelihood ratios to explore neonatal outcomes by advanced paternal age. A likelihood ratio essentially expresses the odds that a given level of a diagnostic test result would be expected in a patient with the target disorder compared to a person without the target disorder. The "positive likelihood ratio' (LR+) tells us how much to increase the probability of disease if the test is positive, and is used to 'rule in' disease. Generally, a LR+> 10 is considered useful to rule in disease. The 'negative likelihood ratio' (LR-) tells us how much to decrease the probability of disease if the test is negative, and is used to rule out disease. Generally, a LR - of $<0.1$ is considered useful to rule out disease.

\section{RESULTS}

The study covered 218 pregnant women that included 137 (63.72\%) pregnant women who delivered a live AGA/LGA baby and 78 (36.28\%) pregnant women who delivered a live small for gestational age baby. The mean \pm SD age of pregnant women in the study was $27.48 \pm 5.57$ years (range 18.00 to 43.00 years, median 26.00 years). A total of 102 (46.79\%) women were primigravid and $126(58.06 \%)$ women were nulliparous. Thirty two (14.68\%) women were obese based on a BMI of $\geq 30$. The mean BMI of women included in the study was $24.76 \pm 5.02$. The mean birthweight (overall) was $2,710.88$ $\pm 547.55 \mathrm{gm}$ (median 2,730.00, range 1,070 to $3,850 \mathrm{gm}$ ). Nineteen $(0.09 \%)$ babies had a birthweight $<2000$ gm including $3(0.01 \%)$ babies with a birthweight $<1500 \mathrm{gm}$.

Compared to women who delivered an AGA/LGA baby, women who delivered a SGA baby were significantly younger (ANOVA $\mathrm{F}=8.77, \mathrm{p}=0.003$ ), were more likely to be nulliparous $\left(\chi^{2}\right.$ test, $\left.\mathrm{p}=0.003\right)$, more likely (Fishers exact test, $\mathrm{p}=0.001)$ to have had a preterm delivery $(<37$ weeks of gestation) and less likely ( $\chi^{2}$ test, $\left.\mathrm{p}=0.001\right)$ to have diabetes (Table 1).

Table 2 shows the frequency distribution of adverse neonatal outcomes in this population. Twenty-six (12.09\%) neonates 
Table 1: Comparison of characteristics of pregnant women included in the study

\begin{tabular}{lccc}
\hline Characteristics & $\begin{array}{c}\text { Delivered AGA/LGA } \\
\text { baby-controls }(n=137)\end{array}$ & $\begin{array}{c}\text { Delivered SGA } \\
\text { baby-cases }(n=78)\end{array}$ & $\begin{array}{c}\text { Statistical test, } \\
p \text {-value }\end{array}$ \\
\hline Woman's mean age \pm SD & $28.15 \pm 5.82$ & $25.90 \pm 4.44$ & ANOVA F = 8.77, $p=0.003$ \\
Degree level education & $100(72.99 \%)$ & $59(75.64 \%)$ & T-test, $p=0.67$ \\
Homemaker & $110(80.29 \%)$ & $63(80.77 \%)$ & $\chi^{2}$ test, $p=0.93$ \\
Mean body mass index & $25.24 \pm 5.48$ & $23.85 \pm 4.09$ & ANOVA F = 3.84, P = 0.05 \\
Obesity (BMI $\geq 30)$ & $25(18.25 \%)$ & $7(8.97 \%)$ & $\chi^{2}$ test, $p=0.06$ \\
Nulliparous & $69(50.36 \%)$ & $55(71.43 \%)$ & $\chi^{2}$ test, $p=0.003$ \\
Primigravid & $76(55.47 \%)$ & $38(48.72 \%)$ & $\chi^{2}$ test, $p=0.34$ \\
Hypertension & $6(4.38 \%)$ & $8(10.26 \%)$ & $\chi^{2}$ test, $p=0.09$ \\
Diabetes & $35(25.55 \%)$ & $6(7.69 \%)$ & $\chi^{2}$ test, $p=0.001$ \\
Heart disease & $1(0.73 \%)$ & $1(1.28 \%)$ & Fisher's exact test, $p=1.00$ \\
Hypothyroidism & $16(11.68 \%)$ & $5(6.41 \%)$ & Fisher's exact test, $p=0.24$ \\
Spontaneous labor & $68(49.64 \%)$ & $35(45.45 \%)$ & $\chi^{2}$ test, $p=0.56$ \\
Preterm delivery & $1(0.73 \%)$ & $9(11.54 \%)$ & Fishers exact test, $p=0.001$ \\
Cesarean section & $63(45.99 \%)$ & $31(39.74 \%)$ & $\chi^{2}$ test, $p=0.38$ \\
\hline
\end{tabular}

required admission to the NICU and $9(4.19 \%)$ neonates had birth malformations.

The mean \pm SD age of spouses was $32.83 \pm 5.98$ years (range 21.00 to 53.00 years, median 31.50 years). The mean \pm SD difference in age between spouses was $5.35 \pm$ 3.02 years (median 5.00 years, range 6.00 to 16.00 years). Only $3(0.01 \%)$ women were aged older to their spouse in this study population. The mean age \pm SD of spouses for women who delivered a SGA baby (30.65 \pm 4.63 years) was significantly lower compared to spouses of women (33.85 \pm 6.24 years) who delivered an AGA/LGA baby (ANOVA F $=15.55, \mathrm{p}=0.001)$.

There were $45(20.64 \%)$ fathers with advanced paternal age based on a cutoff of $\geq 40$ years and 73 (33.49\%) fathers with advanced paternal age if the age cutoff was relaxed to $\geq 35$ years. In a bivariate analysis, advanced paternal age (both $\geq 35$ and $\geq 40$ years) was protective for small for gestational age babies. However, in a multivariate regression model that adjusted for maternal age, parity, diabetes and gestational age at delivery (maternal factors that were significantly different between AGA/LGA and SGA babies in bivariate analysis), advanced paternal age was not significantly associated with SGA babies (Table 3 ). Advanced paternal age (both $\geq 35$ and $\geq 40$ years) did not show a clinically meaningful positive or negative likelihood ratio with other adverse neonatal outcomes. Advanced paternal age (both $\geq 35$ and $\geq 40$ years) was not significantly associated with preterm delivery $\left(\chi^{2}\right.$ test $p$-value 0.06 and 0.39 respectively) or with low birthweight (Fishers exact test p-value 0.25 and 1.00 respectively).

Fetal growth measurements based on ultrasound in the first and second trimesters did not show a significant difference based on paternal age $\geq 40$ years (Tables 4 and 5 ) or paternal age $\geq 35$ years (data not shown).

\section{DISCUSSION}

The results of this study do not suggest an association of advanced paternal age with fetal growth or adverse neonatal outcomes. We did not find a significant association for advanced paternal age and SGA babies. Advanced paternal age was not associated with preterm delivery, admissions to the NICU (indicating severe morbidity), jaundice, birth asphyxia or congenital malformations. The results of this study are consistent with previous reports in the literature that do not cite an association of paternal age with the probability of low birthweight, preterm delivery or adverse neonatal outcomes. ${ }^{2,6,17,19-21}$ Reichman and Teitler, however, documented a positive association between paternal age and low birthweight in an urban population in the United States and recommended the need for further research into the role of paternal factors especially environmental and personal risk behaviors. $^{22}$

Standardized clinical protocols and documentation of findings in an electronic database are advantages of the study. The case-control design allowed us to explore for any potential association between advanced paternal age and fetal growth and adverse perinatal outcomes. Random selection of the control population (from the same setting as the case population) that minimizes selection bias further adds to the strength of the study. However, there are a few limitations to the study. We were not able to collect information on potential confounders especially relating to paternal risk behaviors, paternal medical history, and occupational exposure to environmental risks, nutrition and health seeking behaviors as the father was not always available to provide accurate information. Additionally, such information is not always elicited or documented as part of routine antenatal care especially when the conception is spontaneous or natural. These confounders might possibly influence the results of the study although we are not in a position to comment on the directionality or magnitude of any potential influence. The need to explore the effects of confounders is emphasized by our

Table 2: Neonatal outcomes of the study population (218 pregnant women)

\begin{tabular}{lc}
\hline Neonatal outcomes & $N(\%)$ \\
\hline NICU admissions & $26(12.09 \%)$ \\
Jaundice & $28(13.02 \%)$ \\
Birth asphyxia & $7(3.26 \%)$ \\
Birth malformations & $9(4.19 \%)$ \\
Neonatal hypoglycemia & $10(4.65 \%)$ \\
Low birthweight $(<1500 \mathrm{gm})$ & $3(1.40 \%)$ \\
\hline
\end{tabular}


Table 3: Neonatal outcomes by advanced paternal age

\begin{tabular}{lcccccr}
\hline Characteristics & $\begin{array}{c}\text { Paternal } \\
\text { age }\end{array}$ & $N(\%)$ & $\begin{array}{c}\text { Positive likeli- } \\
\text { hood ratio }\end{array}$ & $\begin{array}{c}\text { Negative likeli- } \\
\text { hood ratio }\end{array}$ & $\begin{array}{c}\text { Unadjusted } \\
\text { odds ratio }\end{array}$ & $\begin{array}{c}\text { Adjusted } \\
\text { odds ratio }\end{array}$ \\
\hline SGA baby & $\geq 35$ & $13(16.67 \%)$ & $0.40(0.23,0.68)$ & $1.43(1.2,1.7)$ & $0.28(0.14,0.55)$ & $0.39(0.10,1.49)$ \\
$(\mathrm{n}=78)$ & $\geq 40$ & $5(6.41 \%)$ & $0.24(0.10,0.58)$ & $1.28(1.14,1.44)$ & $0.18(0.07,0.48)$ & $0.12(0.01,2.94)$ \\
NICU admission & $\geq 35$ & $9(34.62 \%)$ & $1.07(0.61,1.89)$ & $0.96(0.72,1.3)$ & $1.11(0.48,2.59)$ & $0.98(0.12,7.75)$ \\
$(\mathrm{n}=26)$ & $\geq 40$ & $5(19.23 \%)$ & $0.98(0.42,2.27)$ & $1.00(0.82,1.23)$ & $0.98(0.36,2.68)$ & $0.83(0.02,32.04)$ \\
Jaundice & $\geq 35$ & $10(35.71 \%)$ & $1.11(0.65,1.91)$ & $0.95(0.71,1.27)$ & $1.18(0.52,2.66)$ & $0.86(0.22,3.35)$ \\
$(\mathrm{n}=28)$ & $\geq 40$ & $7(25.00 \%)$ & $1.34(0.66,2.71)$ & $0.92(0.74,1.16)$ & $1.45(0.58,3.60)$ & $1.74(0.24,12.48)$ \\
Birth asphyxia & $\geq 35$ & $3(42.86 \%)$ & $1.33(0.55,3.2)$ & $0.84(0.44,1.61)$ & $1.58(0.38,6.5)$ & $0.42(0.003,71.82)$ \\
$(\mathrm{n}=7)$ & $\geq 40$ & $2(28.57 \%)$ & $1.49(0.45,4.95)$ & $0.88(0.55,1.42)$ & $1.68(0.01,7.85)$ & $2.0(0.02,160.09)$ \\
Malformations at birth & $\geq 35$ & $2(22.22 \%)$ & $0.67(0.19,2.32)$ & $1.16(0.81,1.67)$ & $0.58(0.01,2.54)$ & $0.19(0.005,7.55)$ \\
$(\mathrm{n}=9)$ & $\geq 40$ & $1(1.11 \%)$ & $0.56(0.09,3.62)$ & $1.11(0.87,1.41)$ & $0.53(0.01,3.32)$ & $0.46(0.06,3.75)$ \\
\hline
\end{tabular}

Table 4: Fetal growth parameters based on ultrasound in the first trimester by advanced paternal age ( $\geq 40$ years)

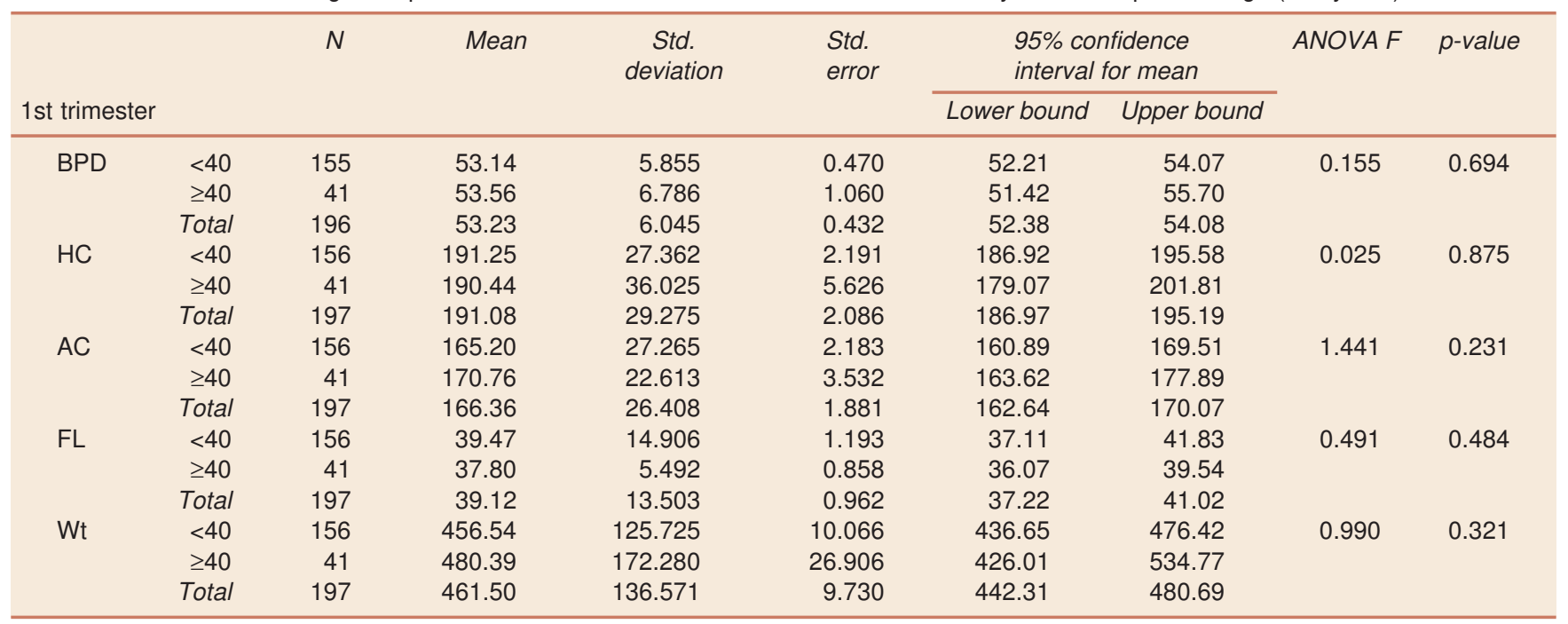

BPD: Biparietal diameter; HC: Head circumference; AC: Abdominal circumference; FL: Femur length; Wt; Weight in grams

Table 5: Fetal growth parameters based on ultrasound in the second trimester by advanced paternal age ( $\geq 40$ years)

\begin{tabular}{|c|c|c|c|c|c|c|c|c|c|}
\hline & \multirow[t]{2}{*}{$N$} & & \multirow[t]{2}{*}{ Mean } & \multirow[t]{2}{*}{$\begin{array}{c}\text { Std } \\
\text { deviation }\end{array}$} & \multirow[t]{2}{*}{$\begin{array}{l}\text { Std } \\
\text { error }\end{array}$} & \multicolumn{2}{|c|}{$\begin{array}{l}95 \% \text { confidence } \\
\text { interval for mean }\end{array}$} & \multirow[t]{2}{*}{ ANOVA $F$} & \multirow[t]{2}{*}{$p$-value } \\
\hline & & & & & & Lower bound & Upper bound & & \\
\hline \multirow[t]{3}{*}{ BPD } & $<40$ & 173 & 78.64 & 7.268 & 0.553 & 77.55 & 79.73 & 0.027 & 0.869 \\
\hline & $\geq 40$ & 44 & 78.84 & 6.630 & 0.999 & 76.83 & 80.86 & & \\
\hline & Total & 217 & 78.68 & 7.129 & 0.484 & 77.73 & 79.64 & & \\
\hline \multirow[t]{3}{*}{$\mathrm{HC}$} & $<40$ & 173 & 284.87 & 22.812 & 1.734 & 281.45 & 288.30 & 0.280 & 0.597 \\
\hline & $\geq 40$ & 44 & 286.89 & 21.332 & 3.216 & 280.40 & 293.37 & & \\
\hline & Total & 217 & 285.28 & 22.486 & 1.526 & 282.27 & 288.29 & & \\
\hline \multirow[t]{3}{*}{$A C$} & $<40$ & 173 & 258.04 & 31.977 & 2.431 & 253.24 & 262.84 & 3.587 & 0.060 \\
\hline & $\geq 40$ & 44 & 268.07 & 28.744 & 4.333 & 259.33 & 276.81 & & \\
\hline & Total & 217 & 260.07 & 31.545 & 2.141 & 255.85 & 264.29 & & \\
\hline \multirow[t]{3}{*}{$\mathrm{FL}$} & $<40$ & 173 & 60.49 & 17.638 & 1.341 & 57.84 & 63.14 & 0.017 & 0.896 \\
\hline & $\geq 40$ & 44 & 60.14 & 6.102 & 0.920 & 58.28 & 61.99 & & \\
\hline & Total & 217 & 60.42 & 15.974 & 1.084 & 58.28 & 62.56 & & \\
\hline \multirow[t]{3}{*}{ Wt } & $<40$ & 173 & 1660.76 & 471.798 & 35.870 & 1589.96 & 1731.57 & 1.625 & 0.204 \\
\hline & $\geq 40$ & 44 & 1764.68 & 524.688 & 79.100 & 1605.16 & 1924.20 & & \\
\hline & Total & 217 & 1681.83 & 483.538 & 32.825 & 1617.14 & 1746.53 & & \\
\hline
\end{tabular}

BPD: Biparietal diameter; HC: Head circumference; AC: Abdominal circumference; FL: Femur length; Wt: Weight in grams

finding of a protective, although nonsignificant effect of advanced paternal age with SGA.

Much of the research on reduced growth has focused on genetic, maternal and fetal factors. These include maternal factors, like malnourishment, anemia, chronic diseases like hypertension, advanced diabetes with complications, renal diseases, cardiac or respiratory diseases, infections during pregnancy, substance abuse and smoking. Fetal factors include multiple gestations, fetal infections, birth defects and chromosomal abnormalities. Placenta- 
uterine factors include decreased blood flow in the uterus and placenta, placental abruption, placenta previa and infections affecting the tissues around the uterus. Paternal factors assume significance as age is no longer a barrier for conception especially with the advancements in assisted reproductive technologies.

To conclude, the results of this study do not suggest an association of advanced paternal age with fetal growth or adverse perinatal outcomes. Although the study has adequate power, further research through a prospective cohort study design that controls for potential confounders is necessary to elucidate associations of advanced paternal age with multiple outcomes.

\section{REFERENCES}

1. Selvin S, Garfinkel J. Paternal age, maternal age and birth order and the risk of a fetal loss. Hum Biol 1976;48:223-30.

2. Olshan AF, Schnitzer PG, Baird PA. Paternal age and the risk of congenital heart defects. Teratology 1994;50:80-84.

3. Stene J, Fischer G, Stene E, Mikkelsen M, Petersen E. Paternal age effect in Down's syndrome. Ann Hum Genet 1977;40: 299-306.

4. Jayasekara R, Street J. Parental age and parity in dyslexic boys. J Biosoc Sci 1978;10:255-61.

5. Krishnaswamy S, Subramaniam K, Indran H, et al. Paternal age and common mental disorders. World J Biol Psychiatry 2009:16.

6. Abel EL, Kruger M, Burd L. Effects of maternal and paternal age on Caucasian and native American preterm births and birth weights. Am J Perinatol 2002;19:49-54.

7. Astolfi P, De Pasquale A, Zonta LA. Paternal age and preterm birth in Italy, 1990 to 1998. Epidemiology 2006;17:218-21.
8. Olshan AF, Ananth CV, Savitz DA. Intrauterine growth retardation as an endpoint in mutation epidemiology: An evaluation based on paternal age. Mutat Res 1995;344:89-94.

9. Zhu JL, Madsen KM, Vestergaard M, Basso O, Olsen J. Paternal age and preterm birth. Epidemiology 2005;16:259-62.

10. Miozzo M, Simoni G. The role of imprinted genes in fetal growth. Biol Neonate 2002;81:217-28.

11. Schwartz D, Mayaux MJ, Spira A, et al. Semen characteristics as a function of age in 833 fertile men. Fertil Steril 1983;39: 530-35.

12. Crow JF. The origins, patterns and implications of human spontaneous mutation. Nat Rev Genet 2000;1:40-47.

13. Reichman NE, Teitler JO. Paternal age as a risk factor for low birthweight. Am J Public Health 2006;96:862-66.

14. Wilcox MA, Newton CS, Johnson IR. Paternal influences on birthweight. Acta Obstet Gynecol Scand 1995;74:15-18.

15. Klebanoff MA. Paternal and maternal birthweights and the risk of infant preterm birth. Am J Obstet Gynecol 2008;198:58-63.

16. Strobino BR, Kline J, Stein Z. Chemical and physical exposures of parents: Effects on human reproduction and offspring. Early Hum Dev 1978;1:371-99.

17. Shah Prakesh S. On behalf of the knowledge synthesis group on preterm/low birthweight births. Paternal factors and low birthweight, preterm, and small for gestational age births: A systematic review. AJOG 2010;103-23.

18. Jager S. Sperm nuclear stability and male infertility. Arch Androl 1990;25:253-59.

19. Nahum GG, Stainslow H. Relationship of paternal factors to birth weight. J Reprod Med 2003;48:963-68.

20. Basso O, Wilcox AJ. Paternal age and delivery before 32 weeks. Epidemiology 2006;17:475-78.

21. Tough SC, Faber AJ, Svenson LW, Johnston DW. Is paternal age associated with an increased risk of low birthweight, preterm delivery, and multiple birth? Can J Public Health 2003;94:88-92.

22. Reichman NE, Teitler JO. Paternal age as a risk factor for low birthweight. Am J Public Health 2006;96:862-66. 